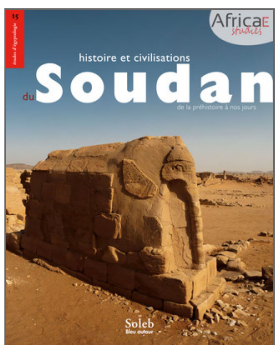

Histoire et civilisation du Soudan

De la préhistoire à nos jours

\title{
Cinquante années
}

\section{Abdel Rahman Ali Mohamed}

DOI : 10.4000/books.africae. 2742

Éditeur : Africae, Soleb, Bleu autour

Lieu d'édition : Paris, Khartoum

Année d'édition : 2017

Date de mise en ligne : 17 janvier 2022

Collection : Africae Studies

EAN électronique : 9782493207074

\section{OpenEdition}

\section{Books}

http://books.openedition.org

\section{Référence électronique}

RAHMAN ALI MOHAMED, Abdel. Cinquante années In : Histoire et civilisation du Soudan : De la préhistoire à nos jours [en ligne]. Paris, Khartoum : Africae, 2017 (généré le 28 janvier 2022). Disponible sur Internet : <http://books.openedition.org/africae/2742>. ISBN : 9782493207074. DOI : https://doi.org/ 10.4000/books.africae.2742. 


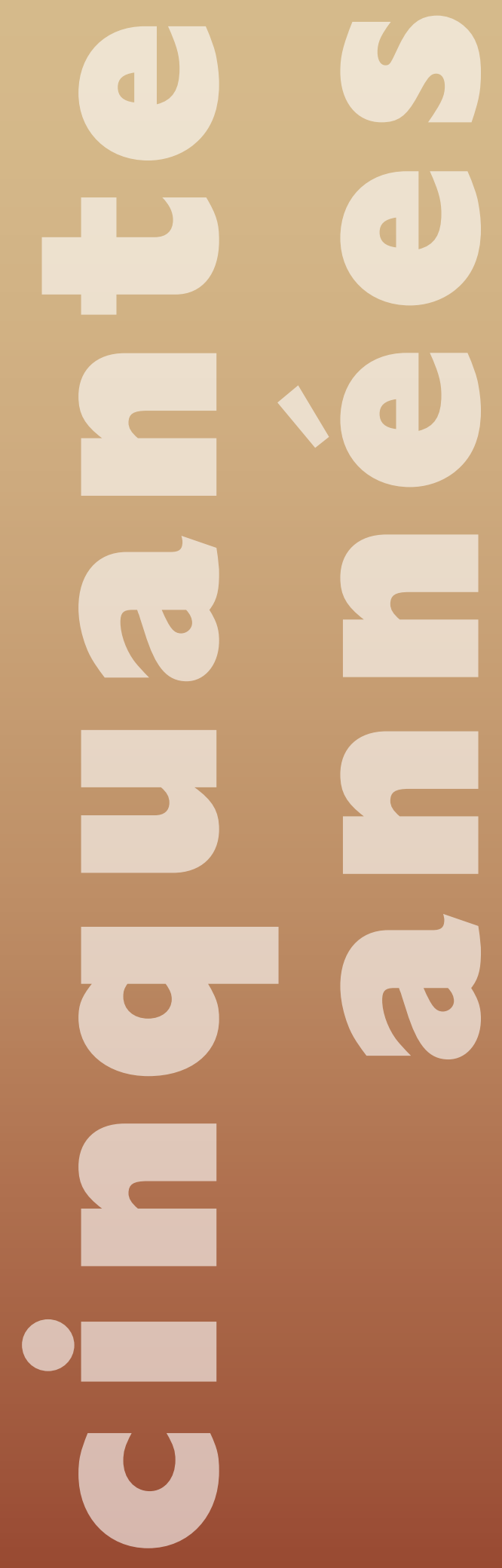


La National Corporation for Antiquities and Museums a été créée dès 1905 et était alors placée sous l'égide du ministère de l'Éducation. En 1971, concomitamment à l'inauguration du musée national du Soudan, ses statuts ont changé. Désormais sous l'autorité du ministère de la Culture, la NCAM, riche d'une longue et féconde histoire, est responsable de l'archéologie et des musées du Soudan.

\section{une triple mission}

La NCAM a une triple mission et comprend autant de départements spécialisés. La première de ces missions concerne les activités de terrain: les surveys et les missions archéologiques; la supervision des fouilles entreprises par les missions soudanaises ou étrangères; la garde et la protection des sites sur lesquels se trouvent des vestiges archéologiques remarquables et de ceux sur lesquels des fouilles sont entreprises.

Cette mission est accomplie sous la direction des officiers et des inspecteurs des antiquités. Les sites sont surveillés par des gardes salariés par la NCAM et par la police des antiquités et du tourisme. (Citons, parmi les nombreux chantiers de fouilles en activité, Kerma-Doukki Gel, Saï, Sedeinga, el-Hassa, el-Kadada, Kadrouka... et, parmi les vestiges archéologiques remarquables, Méroé, Soleb, Kerma, Moussawarat es-Soufra, le Gebel Barkal, Nouri, Kourrou...) 
La seconde mission a trait, notamment, au musée national du Soudan, qui est une structure intégrée à la NCAM. Les objets provenant des fouilles et entrant dans ses collections permanentes sont, le plus souvent, conservés pour étude dans les réserves; dès lors qu'ils sont particulièrement remarquables, ils peuvent être exposés de manière permanente ou présentés lors d'expositions temporaires; ils sont, enfin, susceptibles d'être mis en dépôt dans les différents musées régionaux ou d'être prêtés à l'étranger pour des expositions prestigieuses.

Cette mission englobe également l'administration des musées régionaux et la création de nouveaux musées dans toutes les provinces soudanaises. Ces créations visent à unifier les collections nationales et régionales en un seul "contexte», de manière à en assurer la cohérence sociale et à promouvoir, sous l'égide de la NCAM, un tourisme «responsable». Ces musées ont vocation à être spécialisés, par discipline: musée de la Monnaie, musée de l'Agriculture... La NCAM administre actuellement treize musées. Parmi les principaux, citons:

— Le musée d'Ethnographie, ouvert en 1956 dans les anciens locaux du club de l'armée britannique, sur la rue al-Gama’a, à Khartoum;

— Le musée du Khalifa, créé en 1928, à Omdurman, dans la maison occupée par Abdullahi, successeur du Mahdi, non loin de l'emplacement de la tombe de ce dernier; 
— Le musée de Sheikan, ouvert en 1965 à el-Obeid, au Kordofan, pour commémorer la bataille remportée en 1883 par les troupes du Mahdi sur celles du colonel britannique William Hicks, passé à la postérité sous le nom de Hicks Pacha;

— Le palais du sultan Ali Dinar, dernier souverain du Darfour, à el-Fashir, devenu ensuite résidence des gouverneurs britanniques et transféré à la NCAM en 1971;

— Le musée de Kerma, inauguré en 2008, à la suite des fouilles de Charles Bonnet et qui présente un panorama de l'archéologie régionale depuis les premières occupations humaines jusqu’à la période actuelle.

La troisième mission de la NCAM concerne la conservation et la restauration des antiquités et des œuvres d'art. Le département des Restaurations travaille avec les missions archéologiques pour assurer la préservation des objets mis au jour et est engagé dans un contrôle et une remise en état permanents des sites et des monuments.

Ce département agit également en lien avec celui du musée national et assure la surveillance des objets conservés dans les réserves ou présentés de manière permanente. Il assure également, à la demande, la restauration des œuvres. Il travaille, de plus, en liaison étroite et fréquente avec de nombreuses institutions scientifiques étrangères dont il reçoit souvent les membres venus en mission d'étude. 


\section{l'archéologie au Soudan}

La NCAM est, également, l'héritière d'une longue et riche histoire «archéologique», parfois mouvementée. «Monopolisées» dans les premiers temps par les égyptologues, les «études nubiennes» ont, à la suite des fouilles de sauvetage rendues nécessaires par la création du lac Nasser, connu un développement considérable et sont désormais une discipline à part entière de l'histoire et de l'archéologie.

Après James Bruce, «inventeur» des sources du Nil en 1772, et Burckhardt, venu en 1814, le Français Frédéric Cailliaud accompagne, entre 1820 et 1822, Ismaïl Pacha "général des armées de son père Méhémet

le Soudan Ali». (Admirateur de Bonaparte, le vice-roi souhaite, comme celui-ci, donner une sorte de justification scientifique à son expédition coloniale en s'entourant de savants, le plus souvent français et parfois anglais.)

Il est presque immédiatement suivi par Linant de Bellefonds, qui s'est joint à l'expédition anglaise de Bankes. À Moussawarat es-Soufra, derrière la grande terrasse, sur le mur nord du complexe central, deux inscriptions en français et quasi identiques, perdues au milieu de la savane soudanaise, montrent bien la rivalité entre les deux savants: Louis Linant de Bellefonds, premier des deux explorateurs à avoir visité le site, se dit «mandé par l'Angleterre» et Frédéric Cailliaud, dont l'ironie est perceptible, déclare, lui, avoir été «mandé de la France». 
Quelques années plus tard, vient le temps des aventuriers indépendants, dont le plus connu est un médecin italien: Giuseppe Ferlini. Venu en 1830 comme chirurgien-major de l'armée, mais bien décidé à trouver des trésors et à faire fortune, il déserte. À Méroé, il fait exploser sans scrupule certaines des pyramides avec de la poudre à canon. Dans la tombe de la reine Amanishakhéto, il trouve un petit chaudron rempli de bijoux qu'il vendra avec beaucoup de mal. Ces bijoux sont, en effet, très étranges aux yeux des spécialistes de l'art égyptien. Il faudra que l'égyptologue allemand Lepsius, de retour du Soudan, prouve qu'il s'agit bien de trésors méroïtiques et non de faux grossiers. Ces magnifiques bijoux sont alors achetés pour moitié par le roi de Prusse et pour moitié par le roi de Bavière et sont donc, aujourd'hui, répartis entre les musées de Berlin et de Munich.

En 1842, une expédition est placée par Frédéric-Guillaume IV,

roi de Prusse, sous la direction de Carl Richard Lepsius, un des meilleurs égyptologues de son temps, parfois présenté comme le successeur de Champollion. Lepsius parcourt la vallée du Nil en Égypte et il remonte le fleuve jusqu’au cœur du Soudan, allant jusqu’à Méroé, et même un peu au-delà. (Champollion était venu de 1828 à 1829 à la tête d'une expédition envoyée par Charles X, roi de France, mais il s'était arrêté à la seconde cataracte.) Lepsius collecte un nombre impressionnant d'objets, ce qui explique que le musée de Berlin soit le plus riche d'Europe en antiquités soudanaises. 
En 1846, avec l'accord de Méhémet Ali, vice-roi d'Égypte, un bâtiment revient chargé de 249 caisses contenant 1500 objets. La publication, entre 1849 et 1859 , des douze volumes des Denkmäler aus Aegypten und Nubien («Monuments d'Égypte et de Nubie») va permettre aux savants de redécouvrir la région en présentant quantité de textes et de dessins beaucoup plus précis que ceux publiés auparavant. (Cailliaud en effet ne connaissait pas l'égyptien alors que Lepsius était excellent égyptologue, d'où de meilleurs fac-similés.) Avec ces fameux Denkmäler, les savants disposent véritablement d'un matériel de grande qualité scientifique.

Ladministration coloniale est soutenue par les Occidentaux, avec l'aide d'administrateurs souvent chrétiens, venus notamment du Liban ou de Syrie, mais également de Grande-Bretagne. Le poids des impôts levés sur le pays et l'occidentalisation croissante des Turcs explique la réaction des Soudanais contre ce gouvernement et l'établissement d'un pouvoir islamique pendant une douzaine d'années.

Muhammad Ahmad ibn Abd Allah Al-Mahdi se révolte en 1881 et prend Khartoum le 26 janvier 1885, malgré la défense acharnée de Charles Gordon (qui est tué durant le combat). Le Mahdi meurt le 22 juin 1885 et son bras droit, Abdullahi, lui succède. Pendant la Mahdiyya (1885-1898), l'archéologie se trouve évidemment au point mort et toutes les missions scientifiques sont interrompues. 
En 1884-1885, Horatio Herbert Kitchener avait fait partie de l'expédition qui avait échoué à sauver le général Charles Gordon. Sous sa direction, entre 1896 et 1898 , les Anglais s'emparent progressivement du Soudan et parachèvent leur conquête lors de la bataille d'Omdourman, au cours de laquelle plus de 15000 mahdistes, équipés de lances, sont tués.

Kitchener met dès lors en place un nouveau pouvoir colonial, le "condominium anglo-égyptien", associant les deux pays. Les archéologues commencent à revenir. Ils sont essentiellement britanniques dans un premier temps, puis allemands. Les Français, quant à eux, se sont passionnés pour l'Égypte à la suite des découvertes de Champollion et sont en lutte avec les Anglais pour la constitution d'un Empire colonial: il faudra attendre longtemps pour qu'ils s'intéressent au Soudan. Tout cela explique que le British Museum possède des collections d'antiquités soudanaises importantes, mais pas le musée du Louvre.

Entre 1898 et 1956, l'administration britannique est la première à développer la gestion du patrimoine archéologique soudanais. Les Britanniques mettent également en œuvre, dès 1905, la première législation sur les antiquités, à la base de la loi actuelle et, la même année, la National Corporation for Antiquities and Museums voit le jour. En 1939, enfin, un poste spécifique est créé, celui de Commissioner for Archaeology, assuré en premier lieu par Anthony John Arkell. 


\section{des années de coopération scientifique}

Peu après la proclamation de l'indépendance, le 19 décembre 1955, les autorités soudanaises confient à un Français, Jean Vercoutter, la direction de la gestion des antiquités. C'est lui qui assurera la transition menant, en 1971, à l'occasion de l'inauguration du musée national du Soudan, à la redéfinition des missions de la National Corporation for Antiquities and Museums. Homme d'expérience au Soudan, il fouille dès 1953 la ville fortifiée de Kor, en Basse Nubie, datant du Moyen Empire, et lance en 1954 les premiers travaux sur l'île de Saï. Il est également très impliqué dans le sauvetage des antiquités menacées par le haut barrage d'Assouan. (Annoncé en 1954 par Gamal Abdel Nasser et mis en eau en 1973, ce barrage a amené la création du lac Nasser qui a noyé une large partie de la Nubie, forçant les populations à l'exil et entraînant la disparition d'un vaste patrimoine archéologique.)

Jean Vercoutter supervise plusieurs missions de prospection des zones en danger, la couverture photographique associée, et fait appel à la communauté internationale pour intégrer le Soudan à la grande campagne de l'Unesco. Nommé professeur à l'université Charles-de-Gaulle-Lille 3 en 1961, il engage l'institution dans la campagne de sauvetage des monuments de la Nubie. À l'époque, cette implication fait de l'université le centre névralgique de la recherche française en archéologie soudanaise, via l'association d'une unité du CNRS à l'institution et au lancement de deux fouilles archéologiques 
sur les sites d'Akcha et de Mirgissa. En 1969, la fin de la campagne de Nubie permet à Jean Vercoutter de concentrer ses travaux à Saï, mission qu'il dirigera jusqu'en 1981 .

Dans le même temps, la section française de la direction des Antiquités du Soudan (Sfdas) est fondée en 1967, toujours à l'initiative de Jean Vercoutter. Régie par «l'Accord de coopération culturelle et technique entre le gouvernement de la République démocratique du Soudan et le gouvernement de la République française», signé à Khartoum le 22 décembre 1969, il y a cinquante ans, la Sfdas assure la coopération de la France et du Soudan, tant dans le domaine des fouilles que dans celui de leur publication. La muséographie, la conservation du patrimoine et l'élaboration de rapports d'activité font également partie de ses missions.

La Sfdas est, depuis la signature de cet accord, intégrée à la National Corporation for Antiquities and Museums — au sein, plus précisément, du musée national du Soudan. Cette position privilégiée lui permet d'apporter un soutien aux missions françaises et étrangères travaillant au Soudan et de développer des partenariats scientifique et muséologique. La Sfdas, étant le plus ancien et le plus important institut archéologique permanent au Soudan, occupe dès lors une position de fer de lance de la recherche scientifique française dans le pays. Au partenariat naturel avec la NCAM s'ajoutent des coopérations variées avec les établissements scientifiques français et les universités 
tant soudanaises qu'européennes. Avec l'appui de l'ambassade de France à Khartoum, elle permet à la diplomatie française d'entretenir durablement ses relations avec le Soudan.

Depuis cinquante ans, la Sfdas a été dirigée successivement par André Vila (1969-1975), Francis Geus (1975-1984), Jacques Reinold (19842000), Francis Geus (2000-2004), Vincent Rondot (2005-2009), Claude Rilly (2009-2014) et Vincent Francigny (2014-2019); depuis avril 2019 elle l'est par Marc Maillot qui y fut, auparavant, chercheur. C'est donc cinquante années de travail en commun et d'intégration fructueuse de la Sfdas au sein de la Ncam que nous célébrons cette année; cinquante années de relations fécondes et amicales; cinquante années au cours desquelles de nombreuses découvertes ont été effectuées et ont complété, ou remis en question, nos connaissances dans les domaines de l'archéologie et de l'histoire.

Je suis heureux qu'à l'occasion de ce cinquantenaire sorte la version numérique — diffusée gratuitement à travers le monde — de l'ouvrage paru en 2017 avec l'appui de la NCAM et qui a fait date. Je ne doute pas que réussiront à en être publiées, un jour prochain, les traductions en arabe et en anglais que le Soudan et la communauté scientifique internationale appellent de leurs vœux

Abdel Rahman Ali Mohamed directeur de la National Corporation for Antiquities and Museums 


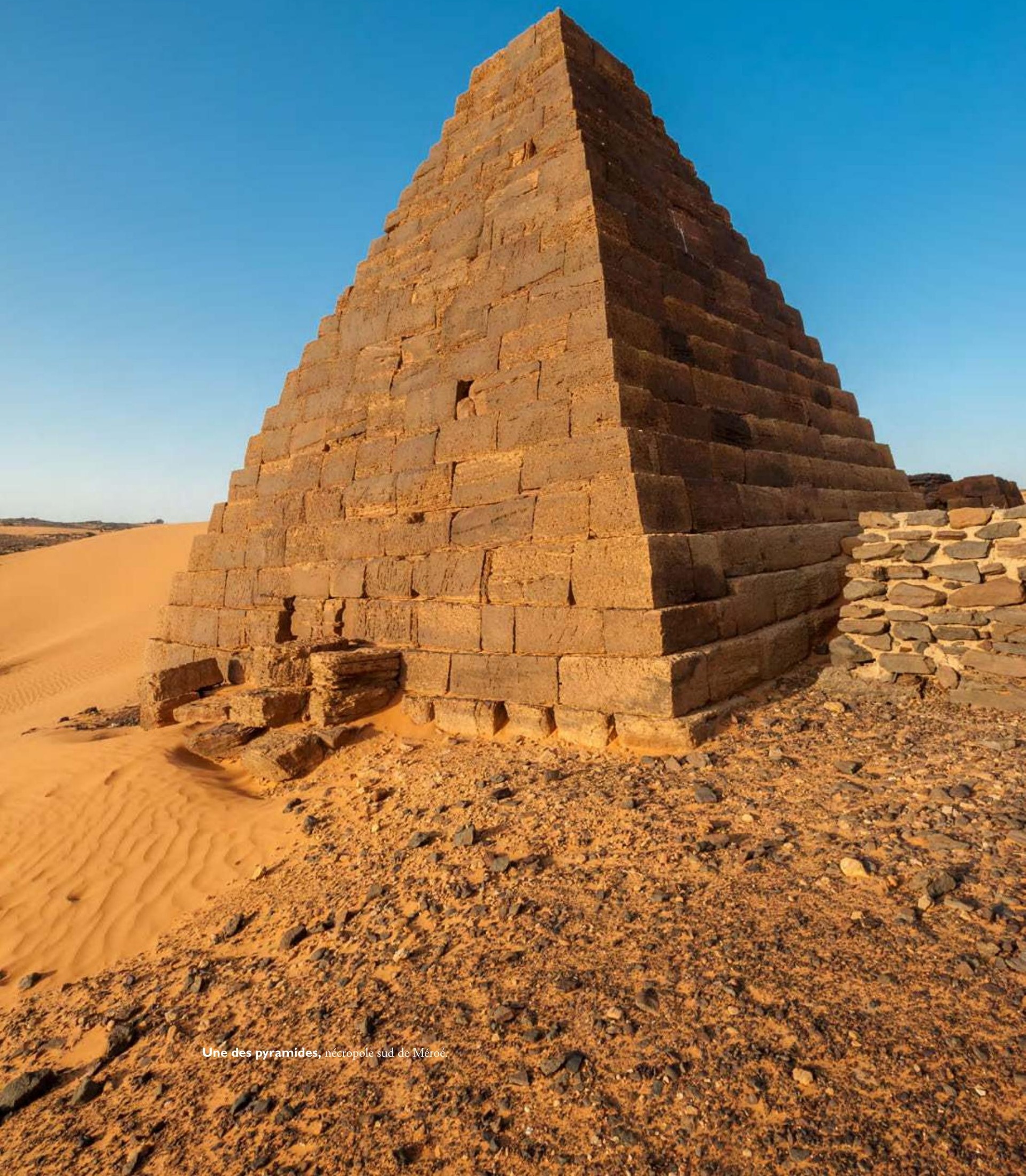

